\title{
Strategic bidding in electricity markets: An agent-based simulator with game theory for scenario analysis
}

Pinto, Tiago; Praca, Isabel; Morais, Hugo; Sousa, Tiago M

Published in:

Integrated Computer-Aided Engineering

Link to article, DOI:

$10.3233 /$ ICA-130438

Publication date:

2013

Link back to DTU Orbit

Citation (APA):

Pinto, T., Praca, I., Morais, H., \& Sousa, T. M. (2013). Strategic bidding in electricity markets: An agent-based simulator with game theory for scenario analysis. Integrated Computer-Aided Engineering, 20(4), 335-346. https://doi.org/10.3233/ICA-130438

\section{General rights}

Copyright and moral rights for the publications made accessible in the public portal are retained by the authors and/or other copyright owners and it is a condition of accessing publications that users recognise and abide by the legal requirements associated with these rights.

- Users may download and print one copy of any publication from the public portal for the purpose of private study or research.

- You may not further distribute the material or use it for any profit-making activity or commercial gain

- You may freely distribute the URL identifying the publication in the public portal 


\title{
Strategic Bidding in Electricity Markets: an Agent-Based Simulator With Game Theory For Scenario Analysis
}

\author{
Tiago Pinto ${ }^{\mathrm{a}}$, Isabel Praça ${ }^{\mathrm{a}}$, Zita Vale $^{\mathrm{a}, *}$, Hugo Morais ${ }^{\mathrm{a}}$, Tiago M. Sousa ${ }^{\mathrm{a}}$ \\ ${ }^{a}$ GECAD - Knowledge Engineering and Decision Support Research Centre - Polytechnic of Porto, Rua Dr. \\ António Bernardino de Almeida, 431, 4200-072 Porto, Portugal
}

\begin{abstract}
Electricity markets are complex environments, involving a large number of different entities, with specific characteristics and objectives, making their decisions and interacting in a dynamic scene. Game-theory has been widely used to support decisions in competitive environments; therefore its application in electricity markets can prove to be a high potential tool. This paper proposes a new scenario analysis algorithm, which includes the application of game-theory, to evaluate and preview different scenarios and provide players with the ability to strategically react in order to exhibit the behavior that better fits their objectives. This model includes forecasts of competitor players' actions, to build models of their behavior, in order to define the most probable expected scenarios. Once the scenarios are defined, game theory is applied to support the choice of the action to be performed. MASCEM (Multi-Agent System for Competitive Electricity Markets) is a multi-agent electricity market simulator that models market players and simulates their operation in the market. The scenario analysis algorithm has been tested within MASCEM and our experimental findings with a case study based on real data from the Iberian Market are presented and discussed.
\end{abstract}

Keywords: Decision Making; Electricity Markets; Intelligent Agents; Game Theory; Multiagent Systems; Scenario Analysis

\section{Introduction}

All over the world electricity restructuring placed several challenges to governments and to the companies that are involved in generation, transmission and distribution of electrical energy. Potential benefits, however, depend on the efficient operation of the market. Definition of the market structure implies a set of complex rules and regulations that should not encourage strategic behaviors that might reduce market performance and lead to market power [23]. Interveners need to rethink their strategies and behavior.
Several market models exists, with different rules and performances creating the need to foresee market behavior, regulators want to test the rules before they are implemented and market players need to understand the market so they may reap the benefits of a well-planned action .

Usually, during the recent years, electricity market players use rather simple strategic behaviors. Most entities keep their biddings constant along the time, while others base their proposed prices in the generation costs of their installations. The most elaborated strategic behaviors go no further than performing

\footnotetext{
* The corresponding author - Zita Vale is with GECAD - Knowledge Engineering and Decision-Support Research Group of the School of Engineering - Polytechnic Institute of Porto (ISEP/IPP), Rua Dr. António Bernardino de Almeida, 431, 4200-072 Porto, Portugal. Telephone: +351228 340 511, Fax: +351228 321159 (e-mail: zav@isep.ipp.pt)
} 
simple averages or regressions of the historic market prices. This matter, an highly unexplored and unimplemented issue, of huge importance for the maximization of players profits, supports the need for the development of proper market acting strategies.

The main contribution of this work is to complement the Multi-Agent Simulator for Electricity Markets (MASCEM) [19, 25] simulator. MASCEM is a modeling and simulation tool that has been developed by this team for the purpose of studying complex restructured electricity markets operation. MASCEM's ability to model the most relevant market players and negotiation mechanisms provides the means for an adequate development and study of models and techniques to support market players' actions in the best possible way. It provides market players with simulation and decision-support resources, being able to give them competitive advantage in the market.

The contribution is provided through the development of a new computational model, implemented to support the development of dynamic pricing strategies, taking advantage of the interactive environment between market agents and on the gathered knowledge during market participation. The methodology is characterized as a scenario analysis algorithm able to support players' strategic behavior. The proposed model includes four innovative components which arise as separate, however, complementary contributions: (i) scenarios definition, concerning the automatic creation of distinct market scenarios based on different perspectives and potential states of the electricity market evolution along the time; (ii) players profiles definition, which is an independent computational model directed to the creation of competitor players' models, in what concerns their characteristics and expected behavior, performing analysis and forecasts of their current and past observed actions and continuously gathered information; (iii) possible actions definition, aiming at establishing a set of coherent and realistic possibilities of actions for the supported player to take on an electricity market environment, taking into account each current context (concerning market and competitor players' states at each point in time); (iv) adaptation of the gametheory concept $[5,16]$ to the electricity market negotiation environment, both concerning bi-lateral and multi-lateral negotiations, which is a major contribution by itself, in a sense that this adaptation concerns such a dynamic and specific context, with so many particularities and constraints.

After this introductory section, Section 2 introduces the theme of multi-agent simulation in electricity markets, outlining the main features of MASCEM, providing an essential insight of this simulator, contributing to an adequate understanding of the simulated multi-agent environment, in order to properly expose the advantages of the proposed work; Section 3 explores the proposed computational model, including the game theory approach for scenario analysis; Section 4 presents a case study based on real electricity market data, testing the proposed models and comparing its results with the results for the same scenario using other two well established methodologies for decision support of players acting in electricity markets. Finally Section 5 presents the most relevant conclusions and contributions of this paper.

\section{Multi-Agent simulation of competitive electricity markets}

The employment of simulation tools is a very adequate way to find market inefficiencies or to provide support for market players' decision. Multi-agent based simulation is particularly well fitted to analyze dynamic and adaptive systems with complex interactions among constituents $[2,6,20]$. With multi-agent simulation tools individual behaviors, as well as system behavior and how individual behaviors affect it, may be studied in a model that may be enlarged dynamically to accomplish new rules or participants.

Indeed several multi-agent tools have been fruitfully applied to the study of restructured wholesale power markets $[2,6,14,15,19,20]$. Some of the most relevant tools in this domain are:

- Electricity Market Complex Adaptive System (EMCAS) [14]: software agents with negotiation competence use strategies based on machinelearning and adaptation to simulate Electricity Markets.

- Agent-based Modelling of Electricity Systems (AMES ) [15]: open-source computational laboratory for studying wholesale power markets, restructured in accordance with U.S. Federal Energy Regulatory Commission (FERC); It uses an agent-base test bed with strategically learning electric power traders to experimentally test the extent to which commonly used seller market power and market efficiency measures are informative for restructured wholesale power markets. 
MASCEM was presented to the scientific community in 2003 [19], combining agent based-modeling and simulation. In its initial form MASCEM provided the modeling of the most relevant entities that participate in electricity markets, as well as some of the most common market mechanism found worldwide. One of MASCEM's objectives is to be able to simulate as many market models and players types as possible so it can reproduce in a realistic way the operation of real electricity markets. This enables it to be used as a simulation and decision-support tool for short/medium term purposes but also as a tool to support long-term decisions, such as the ones taken by regulators. MASCEM includes several negotiation mechanisms usually found in electricity markets [22]. It can simulate several types of markets, namely: pool markets, bilateral contracts, balancing markets and forward markets. This implies that each agent must decide whether to, and how to, participate in each market type.

In 2011 a new enhanced version of MASCEM arose [25], where agents use several distinct strategies when negotiating in the market and learning mechanisms in order to best fulfill their objectives. Although MASCEM's purpose is not to explicitly search for equilibrium points, but to help understand the complex and aggregate system behaviors that emerge from the interactions of heterogeneous individuals, agents learn and adapt their strategies during a simulation, thus possibly converging toward equilibrium.

There are also several entities involved in the negotiations in the scope of electricity markets; MASCEM multi-agent model represents all the involved entities and their relationships. MASCEM model includes: a Market Facilitator Agent, Seller Agents, Buyer Agents, Virtual Power Producer (VPP) [26] Agents, VPP Facilitator Agents, a Market Operator Agent and a System Operator Agent.

\subsection{MASCEM strategies for competitor players profiles definition}

In order to build suitable profiles of competitor agents, it is essential to provide players with strategies capable of dealing with the constant changes in competitors' behavior, allowing adaptation to their actions and reactions. For that, it is necessary to have adequate forecasting techniques to analyze the data properly, namely the historic of other agents past actions. The way each agent bid is predicted can be approached in several ways, namely through the use of statistical methods, data mining techniques [7, 21, 24], neural networks (NN) [1, 11], support vector machines (SVM) [27], or several other methods [4, 12]. But since the other agents can be gifted with intelligent behavior as well, and able to adapt to the circumstances, there is no method that can be said to be the best for every situation, only the best for one or other particular case.

To take advantage of the best characteristics of each technique, we decided to create a method that integrates several distinct technologies and approaches. The method consists of the use of several forecasting algorithms, all providing their predictions, and, on top of that, a reinforcement learning algorithm that chooses the one that is most likely to present the best answer. This choice is done according to the past experience of their responses and also to the present characteristics of each situation, such as the week day, the period, and the particular market context in which the players are acting.

The main reinforcement algorithm presents a distinct set of statistics for each acting agent, for their actions to be predicted independently from each other, and also for each period. This means that an algorithm that may be presenting good results for a certain agent in a given period, with its output chosen more often when bidding for this period, may possibly never be chosen as the answer for another period. The tendencies observed when looking at the historic of negotiation periods independently from each other show that they vary much from each other, what suggests that distinct algorithms can present distinct levels of results when dealing with such different tendencies.

The way the statistics are updated, and consequently the best answer chosen, can be defined by the user. MASCEM provides three alternative reinforcement learning algorithms, all having in common the starting point. All the algorithms start with the same value of confidence, and then, according to their particular performance, that value is updated.

All forecasting algorithms also have the option of being attributed a weight value that defines its importance to the system. This means that a strategy that has a higher weight value will detach faster from the rest in case of either success or failure. The three versions are:

- A simple reinforcement learning algorithm, in which the updating of the values is done through a direct decrement of the confidence value $C$ in the time $t$, according to the absolute value of the difference between the prediction $P$ and the real 
value $R$. The updating of the values is expressed by (1).

$$
C_{t+1}=C_{t}-|(R-P)|
$$

- The revised Roth-Erev reinforcement learning algorithm [12] that, besides the features of the previous algorithm, also includes a weight value $W$, ranging from 0 to 1 , for the definition of the importance of past experience. This version is expressed as in (2).

$$
C_{t+1}=C_{t} \times W-|(R-P)| \times(1-W)
$$

- A learning algorithm based on the Bayes theorem of probability [8], in which the updating of the values is done through the propagation of the probability of each algorithm being successful given the facts of its past performance. The expected utility, or expected success of each algorithm is given by (3), being $E$ the available evidences, $A$ an action with possible outcomes $O i$, $U(O i \mid A)$ the utility of each of the outcome states given that action $A$ is taken, $P(O i \mid E, A)$ the conditional probability distribution over the possible outcome states, given that evidence $E$ is observed and action $A$ taken.

$$
E U(A \mid E)=\sum_{i} P\left(O_{i} \mid E, A\right) \times U\left(O_{i} \mid A\right)
$$

The algorithms used for the predictions are:

- A feed-forward neural network trained with the historic market prices, with an input layer of eight units, regarding the prices and powers of the same period of the previous day, and the same week days of the previous three weeks. The intermediate hidden layer has four units and the output has one unit - the predicted bid price of the analyzed agent for the period in question.

- Based on Statistical approaches. There are five strategies in this category:

* Average of prices and powers from the agents' past actions database, using the data from the 30 days prior to the current simulation day, considering only the same period as the current case, of the same week day. This allows us to have a strategy based on the tendencies per week day and per period;

* Average of the agent's bid prices considering the data from one week prior to the current simulation day, considering only business days, and only the same period as the current case. This strategy is only performed when the simulation is at a business day. This ap- proach, considering only the most recent days and ignoring the distant past, gives us a proposal that can very quickly adapt to the most recent changes in this agent's behavior. And is also a good strategy for agents that tend to perform similar actions along the week;

* Average of the data from the four months prior to the current simulation day, considering only the same period as the current case. This offers an approach based on a longer term analysis. Even though this type of strategies, based on averages, may seem too simple, they present good results when forecasting players' behaviors, taking only a small amount of time for their execution;

* Regression on the data from the four months prior to the current simulation day, considering only the same period of the day;

* Regression on the data of the last week, considering only business days. This strategy is only performed when the simulation is at a business day.

- Algorithms based on pattern analysis:

* Sequences in the past matching the last few actions. In this approach are considered the sequences of at least 3 actions found along the historic of actions of this player. The sequences are treated depending on their size. The longer matches to the recent history are attributed a higher importance;

* Most repeated sequence along the historic of actions of this player;

* Most recent sequence among all the found ones.

- Algorithm based on history matching. Regarding not only the player actions, but also the result they obtained. This algorithm finds the previous time that the last result happened, i.e., what the player did, or how he reacted, the last time he performed the same action and got the same result.

- Algorithm returning the most repeated action of this player. This is an efficient method for players that tend to perform recurrent actions.

- Second-Guessing the predictions. Assuming that the players whose actions we are predicting are gifted with intelligent behavior, it is essential to shield this system, avoiding being predictable as well. So this strategy aims to be prepared to situations when the competitors are expecting the actions that the system is performing. 
* Second-Guess: if the prediction on a player action is $P$, and it is expecting the system to perform an action $P 1$ that will overcome its expected action, so in fact the player will perform an action $P 2$ that overcomes the system's expected $P 1$. This strategy prediction is the $P 2$ action, in order for the system to expect the player's prediction;

* Third-Guess: this is one step above the previous strategy. If a player already understood the system's second guess and is expecting the system to perform an action that overcomes the $P 2$ action, than it will perform an action $P 3$ that overcomes the system prediction, and so, this strategy returns $P 3$ as the predicted player action.

\section{Game Theory based Scenario Analysis}

The scenario analysis algorithm supports strategic behavior with the aim of providing complex support to develop and implement dynamic pricing strategies.

Each agent develops a strategic bid, taking into account not only its previous results but also other players' bids and results and expected future reactions. This is particularly suitable for markets based on a pool or for hybrid markets, to support Sellers and Buyers decisions for proposing bids to the pool and accepting or not a bilateral agreement. The algorithm is based on the analysis of several bids under different scenarios. The analysis results are used to build a matrix which supports the application of a decision method to select the bid to propose. Each agent has historical information about market behavior and about other agents' characteristics and past actions. This algorithm's organization is presented in Fig. 1.
- Self Model prediction. Once again if a player is gifted with intelligent behavior, it can perform the same historical analysis on the system's behavior as the system performs on the others. This strategy performs an analysis on its own historic of actions, to predict what itself is expected to do next. From that the system can change its predicted action, to overcome the players that may be expecting it to perform that same predicted action.

Second-Guess the Self Model prediction. The same logic is applied as before, this time considering the expected play resulting from the Self Model prediction.

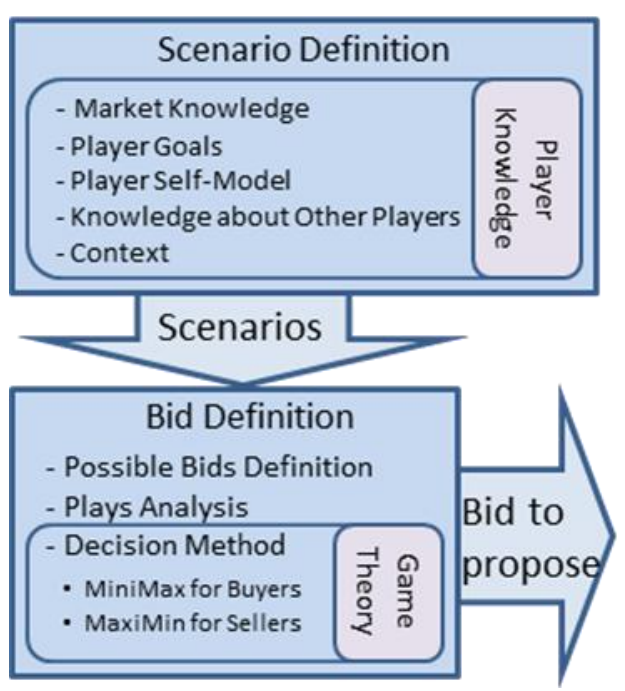

Fig. 1. Scenario Analysis Algorithm.

To get warrantable data, agents using this method perform an analysis of the historical data. With the gathered information, agents can build a profile of other agents including information about their expected proposed prices, limit prices, and capacities. With these profiles, and based on the agent own objectives, several scenarios, and the possible advantageous bids for each one, are defined.

Seller and Buyer agents interact with each other, in MASCEM environment, taking into account that their results are influenced by competitor's decisions. Game theory is well suited for analyzing these kinds of situations $[5,16]$. 


\subsection{Scenario definition}

MASCEM is implemented as a Decision Support tool, so the user should have the flexibility to decide how many and which scenarios should be analyzed. To do so, the user must define the scenarios to be simulated by specifying the price that competitor agents will propose (4):

$$
\begin{gathered}
\text { Price }_{i}=\lambda \times \text { Probable_Price }_{i}+\varphi \times \text { Limit_Price }_{i}, \\
, \lambda+\varphi=1
\end{gathered}
$$

where $\lambda$ and $\varphi$ are scaling factors that can be different for each agent and for each scenario.

Let us suppose that the user selects $\lambda=0$ and $\varphi=1$ for every Seller and $\lambda=1$ and $\varphi=0$ for every Buyer; this means an analysis of a pessimistic scenario. If the user selects $\lambda=1$ and $\varphi=0$ for every agent, then the most probable scenario will be analyzed. Using this formula the user can define for each agent the proposed prices for every scenario that it desires to consider.

The Probable_Price is a predicted value concerning the expected bidding price of each competitor player. This prediction is reached by using the players' profiles definition mechanism, presented in section 2 . This prediction allows the proposed method to use adequate and realistic values when considering other players' actions.

The Limit_Price corresponds to maximum price that can be bided by a seller agent, or the minimum price that can be bided by a buyer agent.

Each scenario considers a fixed number of players, each with constant amounts of power. Only the bidding prices for each player vary from scenario to scenario.

\subsection{Bid definition}

An agent should analyze the income that results from bidding its limit, desired, and competitive prices - those that are just slightly lower (or higher, in the buyers' case) than its competitors' prices.

A play is defined as a pair of bid - scenario, so, the total number of plays to analyze for each player is (5):

$$
n=\text { number_of_bids } \times \text { number_of_scenarios }
$$

and the maximum value it can achieve is (6):

$$
(2 \times n+2) \times 2^{n}
$$

considering that agents only bid their limit or expected prices. However, an agent may bid prices be- tween its limit and expected prices, or even above that limit price. If we consider each agent may bid numprices prices, the number of scenarios becomes equal to npn, and the number of plays to analyze is (7).

$$
\left({\text { numprices } \times n+2) \times \text { numprices }^{n}}^{n}\right.
$$

The user is also allowed to choose the number of bids that will be considered as possibilities for the final bid. In this case, the value of the bids is calculated depending on an interval of values that can also be defined by the user. That interval is always centered on a trusted value, the value of the market price of the same period of the previous day. In this way the considered possible bids are always around that reference value, and their range of variance depends on the bigger or smaller value of the user defined interval.

So, being nb the number of bids defined by the user, int the value defining the interval to be considered, and $\mathrm{mp}$ the market price from the same period of the previous day, the possible bids b1..nb are defined as (8) and (9):

$$
\begin{gathered}
\mathrm{b}_{1}=m p-\frac{i n t}{2} \\
b_{m}=b_{m-1}+\left(\frac{i n t}{n b-1}\right), m \in[2, n b]
\end{gathered}
$$

After defining all the scenarios and bids, market simulation is applied to build a matrix with the expected results for each play.

The matrix analysis with the simulated plays' results is inspired on the game theory concepts for a pure-strategy two-player game, assuming each player seeks to minimize the maximum possible loss or maximize the minimum possible gain.

After each negotiation period, an agent may increase, decrease or maintain its bid, increasing the number of scenarios to analyze. So, after k periods, considering only three possible bid updates, the number of plays to analyze becomes (10):

$$
(n p \times n+2) \times n p^{n} \times 3^{(k-1) \times n}
$$

\section{Game Theory for scenario analysis}

A seller, like an offensive player, will try to maximize the minimum possible gain by using the MaxiMin decision method. A buyer, like a defensive player, will select the strategy with the smallest maximum payoff by using the MiniMax decision method. 
Buyers' matrix analysis leads to the selection of only those situations in which all the consumption needs are fulfilled. This avoids situations in which agents have reduced expenses but cannot satisfy their consumption needs completely.

The state space to be searched is related to the possible plays of other agents, regarding possible bids from one agent. Fig. 2 illustrates this procedure.

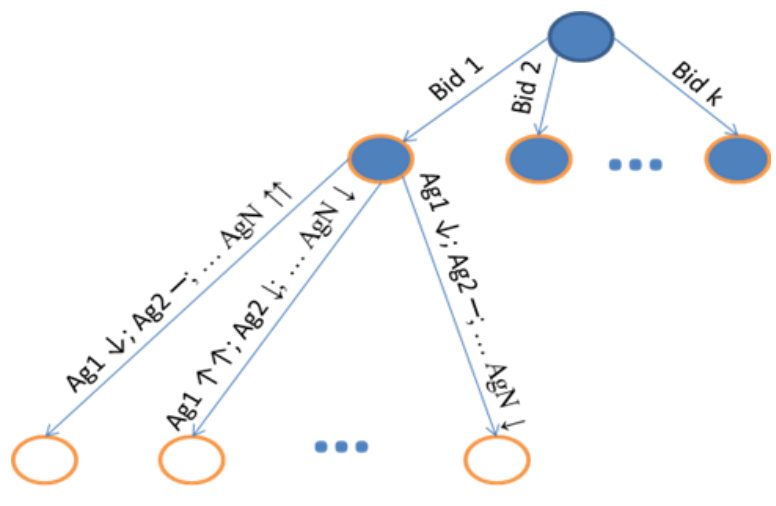

Fig. 2. Game theory for scenario-space search.

Each bid of a specific agent (e.g. $\left.A g_{i}\right)$ is analyzed by considering several possible scenarios, in order to support the decision of this agent. The scenarios are evaluated by considering the prices other agents may propose, regarding the previous proposed prices. It is also considered that each agent may change its price: increasing a lot $(\uparrow \uparrow)$, increasing a little $(\uparrow)$, maintaining (一), decreasing a little $(\downarrow)$, or decreasing a lot $(\downarrow \downarrow)$ its bid price (A little means from 0 to $10 \%$ and a lot from $10 \%$ to $30 \%$ ). Here the concepts of little and lot will consider the historic data of agents' bids and will be converted to variations in cents. It is important to observe that it is impossible to consider all kind of variations, due to the complexity of the problem, as we have seen before. The required time for solving the problem with a large set of combinations would be impractical since a complete market simulation is required for each scenario.

Each leaf node of the tree in Fig. 2 corresponds to a possible scenario. The idea is to evaluate each one of these scenarios and apply a MiniMax or MaxiMin based algorithm to select the safest bid to be offered by agent $A g_{i}$.

Notice that our use of game theory is intended for supporting one specific agent and not for achieving the equilibrium in the market. The idea of the meth- odology proposed in this paper is to provide a specific agent with decision support.

For each simulated scenario (leaf of Fig. 2) we will calculate the price $P_{\text {market }}$ for each MW.h (Megawatt hour), defined as the result of the simulated market. For the support of seller agents the evaluation of the scenario (in profits, $F$ ) is made by the product of the energy sold by the supported agent Agi, Energy_Sold , by the profit, obtained from the difference between $P_{\text {market }}$ and the cost associated to each MW.h sold by Agi, Cost $_{i}$, according to (11):

$$
F=\text { Energy }_{-} \operatorname{sold}_{i} \cdot\left(P_{\text {market }}-\text { Cost }_{i}\right)
$$

Notice that the part of this formula that demands the higher processing cost is the calculation of the value $P_{\text {market }}$, since it implies to run the simulation of the scenario in order to determine the market clearing price.

Additionally, there are two methods for solving problems of equality in the evaluation of scenarios. In case of a seller, the MaxiMin algorithm chooses the bid that offers the maximum gain, from the worst possible scenario. In case of more than one scenario being evaluated with equal value as worst scenario, the options for choosing among them are:

A greedy approach, choosing the scenario, among the equally worst ones, that presents the bid that allows the higher payoff from all the possible bids;

An average of the results of all possible bids for these scenarios, choosing the one that gets the worst average as the worst possible scenario.

The user is able to choose among these two methods for solving the problems of equality. He can also choose a third option that is a mechanism that chooses automatically among these two options, accordingly to the success that each of them is presenting. This mechanism uses a reinforcement learning algorithm, with initial equal values of confidence for the two options. As the time evolves, the values of success of each option are updated, and the one that presents the best confidence in each run, is the one chosen.

The updating of these confidence values is performed by running the two options and saving the answer proposed by each one. Later, after the bid is chosen as the agent's action for the actual market, this method analyzes the market values and checks which of the outputs proposed by each method would have led to the best results.

This procedure is similar to the one used for updating the values of the players' profile definition methodology, by comparing the values proposed by each 
of the algorithms used for forecasting with the actual actions the each player performed in the market.

The scenario analysis algorithm is implemented in JAVA $^{1}$, for a smoother integration with MASCEM simulator. However, for efficiency issues, the majority of data analysis methods, namely the pattern analysis and history matching algorithms for players' profiles definition, are implemented in LPA Prolog ${ }^{2}$. The neural network was developed in MatLab ${ }^{3}$

\section{Experimental findings}

This section presents three simulations undertaken using MASCEM, referring to the same 16 consecutive days, starting from Friday, 15th October, 2010. The data used in this case study has been based on real data extracted from the Iberian market - OMIE [17].

These simulations involve 7 buyers and 5 sellers (3 regular sellers and 2 VPPs). This group of agents was created with the intention of representing the Spanish reality, reduced to a smaller group, containing the essential aspects of different parts of the market, allowing a better individual analysis and study of the interactions and potentiality of each of those actors. This group of agents results from the studies presented in [25].

For these simulations we will consider different biddings for each agent. Seller 2, which will be our test reference, will use the proposed method with different parameters in each of the three simulations. This allows comparing the performance of this method when using distinct parameterizations and taking conclusions on its suitability and the influence of the different parameters presented in Section 3. This section additionally presents the comparison between the results obtained by each of the three considered parameterizations and the results obtained by using two other strategies which are well established and with verified performance and results, in order to determine in what degree the proposed game theory based strategy is best or worst suited for providing decision support to market players. These strategies are:

- The AMES strategy is used by the AMES electricity markets simulator [15] to provide support to the modelled players when bidding in the market. This strategy is based on a study of the

\footnotetext{
${ }^{1}$ http://www.java.com/

2 http://www.lpa.co.uk/

$3 \underline{\text { http://www.mathworks.com/products/matlab }}$
}

efficiency and reliability of the Wholesale Power Market Platform (WPMP), a market design proposed by the U.S. Federal Energy Regulatory Commission for common adoption by all U.S. wholesale power markets $[9,10]$. The AMES strategy was adapted by the authors of this paper in a previous work [18], to suit it to the purposes of asymmetrical and symmetrical pool markets, such as the Iberian Market - OMIE [17]. This strategy uses a reinforcement learning algorithm - the Roth-Erev algorithm [12] to choose from a set of the possible actions (or Action Domain) which is based on the companies' production costs analysis. Additionally, the Simulated Annealing heuristic [3] is implemented to accelerate the convergence process.

- The SA-QL strategy [24] is similar to the AMES strategy in its fundamentals: the use of a reinforcement learning algorithm to choose the best from a set of possible actions. The differences concern two main aspects: the used reinforcement learning algorithm is the Q-Learning [13] algorithm; and the set of different possible bids to be used by the market negotiating agent is determined by a focus on the most probable points of success (in the area surrounding the expected market price). This strategy also uses the Simulated Annealing heuristic to accelerate the process of convergence.

The common parameters in all the simulations using the game theory strategy are: the selection of the automatic mechanism for solving the problems of equality among scenarios; for all seller agents the limit price is fixed as $0 \mathrm{c} € / \mathrm{kWh}$, for it does not make sense to bid negative values; for all buyer agents the limit price is $20 \mathrm{c} € / \mathrm{kWh}$, a high value for allowing the players to consider a good margin of prices. Also, the selected reinforcement learning algorithm for the players' profiles definition has been the revised RothErev, with equal value of the algorithms weight. The past experience weight $\mathrm{W}$ value is set to 0.4 , a small value to grant higher influence to the most recent results, so that it can quickly learn and catch new tendencies in players' actions. For each scenario the scaling factors for competitors' probable price $\lambda$ and limit price $\varphi$, will be equal for every competitor agent, in order to give the same importance to the price forecast of each agent. These scaling factors will only vary from scenario to scenario, but always maintaining the equality among agents. 
The variations introduced in each simulation are:

- In the first simulation Seller 2 will use the scenario analysis method with a small number of considered scenarios and possible bids. This test will allow us to perceive if a restrict group of scenarios, and consequent advantage in processing speed, will be reflected on a big difference in the results quality. For this simulation the number of considered scenarios is 3 , the number of considered bids is 5 , and the interval for the possible bids definition is 8. Considering the 3 scenarios, the first will attribute to all agents $\lambda=1$ and $\varphi=0$; the second $\lambda=0,95$ and $\varphi=0,05$; and the third $\lambda=0,9$ and $\varphi=0,1$. These values give higher importance to the most probable prices, in order to consider the most realistic scenarios.

- In the second simulation Seller 2 will use the scenario analysis method with an intermediate number of considered scenarios and possible bids. The number of considered scenarios is 5, the number of considered bids is 7 , and the interval for the possible bids definition is 8 . Considering the 5 scenarios, the first will attribute to all agents $\lambda=1$ and $\varphi=0$; the second $\lambda=0,95$ and $\varphi=0,05$; the third $\lambda=0,9$ and $\varphi=0,1$; the fourth $\lambda=0,8$ and $\varphi=0,2$; and the fifth $\lambda=0,7$ and $\varphi=0,3$.

- Finally, in the third simulation Seller 2 will use the method with a higher number of considered scenarios and possible bids, in order to obtain a more detailed analysis. The number of considered scenarios is 7 , the number of considered bids is 10 , and the interval for the possible bids definition is 10, granting also a bigger interval for considered bids. Considering the 7 scenarios, the first will attribute to all agents $\lambda=1$ and $\varphi=0$; the second $\lambda=0,95$ and $\varphi=0,05$; the third $\lambda=0,9$ and $\varphi=0,1$; the fourth $\lambda=0,8$ and $\varphi=0,2$; the fifth $\lambda=0,7$ and $\varphi=0,3$; the sixth $\lambda=0,5$ and $\varphi=0,5$; and the seventh $\lambda=0,2$ and $\varphi=0,8$.

After the simulations, the incomes obtained by Seller 2 using the proposed method with each of the three combinations of parameters can be compared. This agent's power production to be negotiated in the market will remain constant at $50 \mathrm{MW}$ for each period throughout the simulations. Regarding the costs of all players, they are defined as null, for facilitating the comparison of the results. The other players' bids are defined as follows:

- Buyer 1 - This buyer buys power independently of the market price. The offer price is 18.30 $\mathrm{c} € / \mathrm{kWh}$ (this value is much higher than average market price).

- Buyer 2 - This buyer bid price varies between two fixed prices, depending on the periods when it really needs to buy, and the ones in which the need is lower. The two variations are 10.00 and $8.00 \mathrm{c} € / \mathrm{kWh}$.

- Buyer 3 - This buyer bid price is fixed at 4.90 $\mathrm{c} € / \mathrm{kWh}$.

- Buyer 4 - This buyer bid considers the average prices of the last 4 Wednesdays.

- Buyer 5 - This buyer bid considers the average prices of the last 4 months.

- Buyer 6 - This buyer bid considers the average prices of the last week (considering only business days).

- Buyer 7 - This buyer only buys power if market prices are lower than average market price.

- Seller 1 - This seller needs to sell all the power that he produces. The offer price is $0.00 \mathrm{c} € / \mathrm{kWh}$.

- Seller 3 - This seller bid considers the average prices of the last 4 months with an increment of $0.5 \mathrm{c} € / \mathrm{kWh}$.

- VPP 1 - Includes 4 wind farms and offers a fixed value along the day. The offer price is 3.50 $\mathrm{c} € / \mathrm{kWh}$.

- VPP 2 - Includes 1 photovoltaic, 1 cogeneration and 1 mini-hydro plants; the offer price is based on the costs of co-generation and the total forecasted production.

Since the reinforcement learning algorithm for the players' profiles definition treats each period of the day as a distinct case, the analysis of the development of the performance must be done for each period individually. Fig. 3 presents the evolution of Seller 2 incomes in the first period of each considered day, along the 16 days, using each of the three considered combinations of parameters.

a)

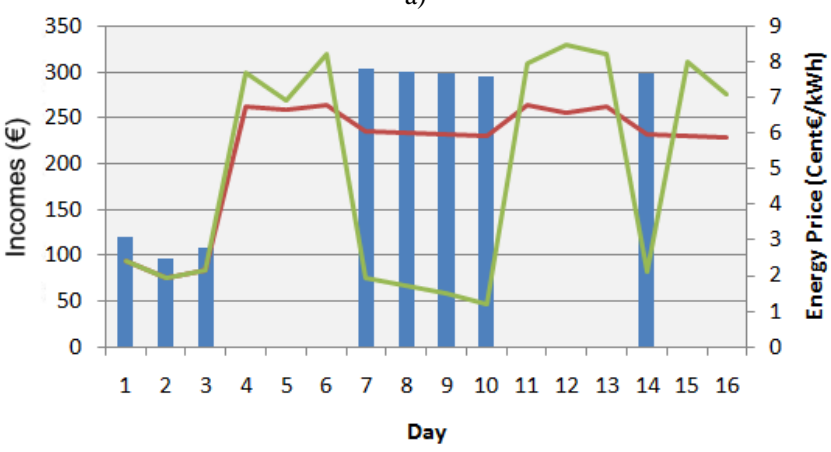


b)

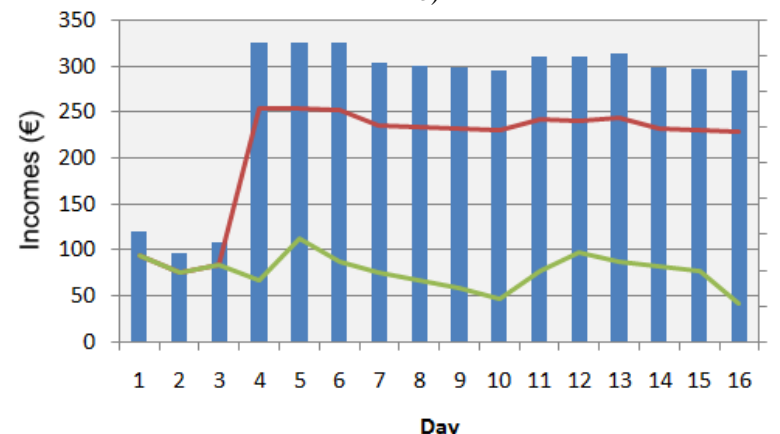

c)

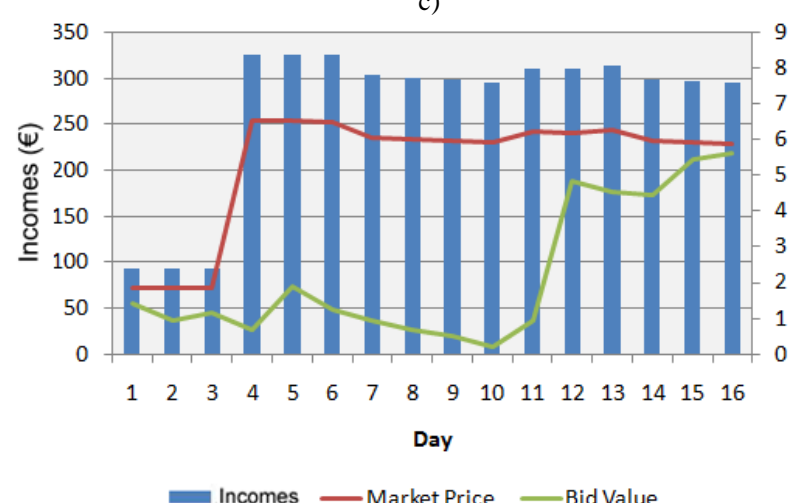

Fig. 3. Incomes obtained by Seller 2 in the first period of the considered 16 days, using: a) the first parameterization, b) the second parameterization, c) the third parameterization.

Fig. 4 presents the results of Seller 2 in the twelfth period of each considered day.

a)

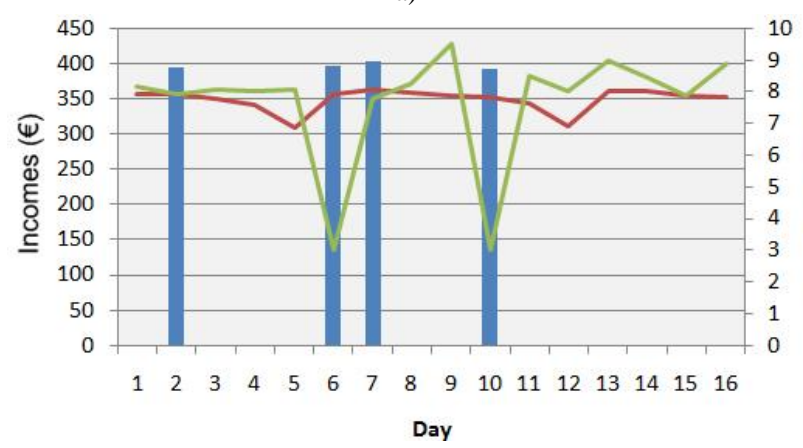

b)

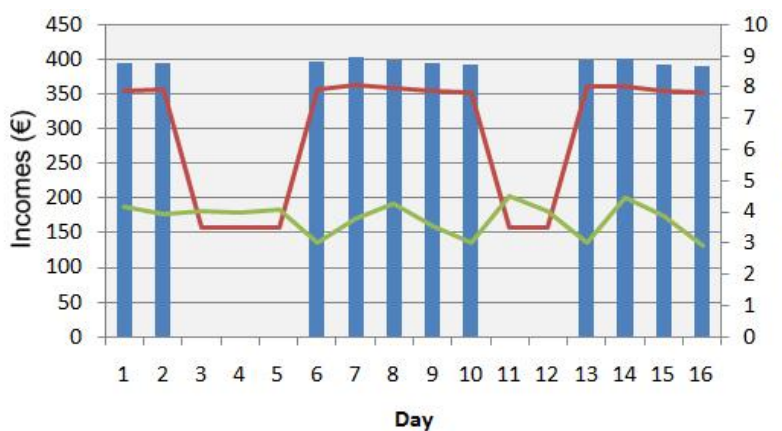

c)

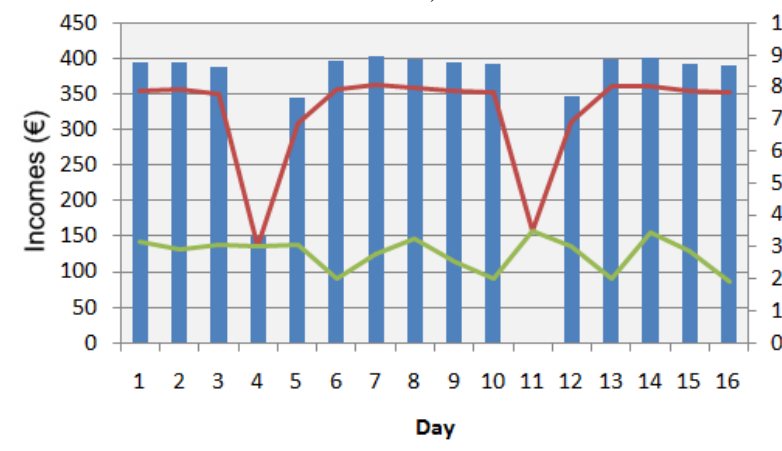

Incomes —Market Price -Bid Value

Fig. 4 Incomes obtained by Seller 2 in the twelfth period of the considered 16 days, using: a) the first parameterization, b) the second parameterization, c) the third parameterization.

Comparing the graphs presented in Fig. 3, it can be concluded that the first simulation was clearly the most disadvantageous for Seller 2 for this period. The second and third simulations present very similar results in what concerns the incomes obtained by this agent in the first period.

The results of the twelfth period show the first parameterization worst results when compared with the other two. However, in this case, the third parameterization clearly obtained better results than the second one. The global results for all periods of the considered 16 days, presented in Fig. 5, support this tendency. Fig. 5 additionally presents the comparison between the three parameterizations of the game theory strategy and the other two strategies' performance: The AMES strategy, and the SA-QL. 


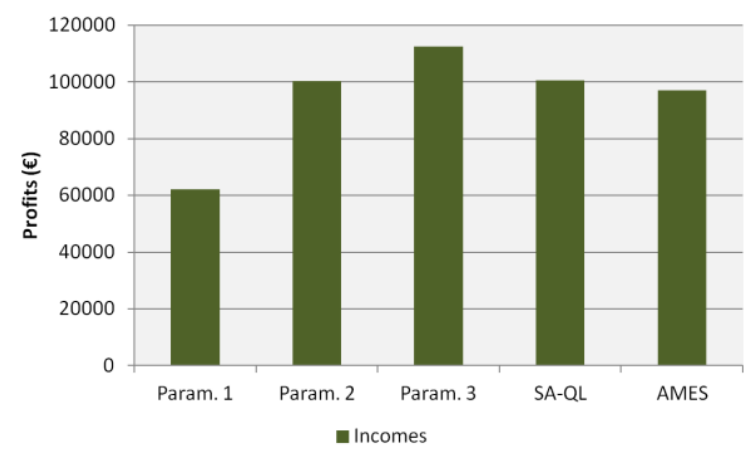

Fig. 5. Total incomes obtained by Seller 2 for the considered 16 days.

From Fig. 5 it is visible that the first parameterization presents a large difference from the other two, and a smaller difference between the results achieved by the second and third parameterizations can be clearly seen. The comparison of the different parameterizations' performances allows taking an important conclusion: when it is required for the simulations to improve the processing times, a criterious reduction of the search space may not represent a significant decrease of the method's effectiveness. As proven by simulation 2, which even though considering fewer scenarios and possible bids than the parameterization of simulation 3 , its results were still acceptable for situations for which the method's processing time is crucial.

Regarding the comparison between the use of the game theory strategy and the other two comparing strategies, it is visible that the first parameterization of the proposed strategy achieves lower results than the two reference strategies. This was expected and it is easily justified by the low number of scenarios and possible bids that this parameterization concerned. The second parameterization achieves very similar results to the ones obtained by the two reference strategies. This means that, even using an intermediate number of scenarios and bids, the proposed game theory strategy is capable of achieving levels of performance that are similar to the results of reference and well established strategies. In what concerns to the third parameterization, it is capable of achieving best results than any of the other strategies, for the considered days. This is a motivating result, suggesting that the proposed method is able to provide better results to a market negotiating player's actions, when the parameters are suitably defined.

\section{Conclusions and Future work}

This paper proposed a computational model for bid definition in electricity markets. The proposed method uses a scenario analysis algorithm based on the principles of game theory to evaluate and preview different scenarios and react strategically. The proposed method is integrated in MASCEM, an electricity market simulator developed by the authors' research centre.

The model proves to be adequate for providing decision support to electricity markets players, allowing an analysis of different scenarios, taking into account the predictions of competitor players' actions.

The results presented in the experimental findings section show that it can achieve good results when using suitable parameterizations, as in simulation 3 . These good results are also shown not to be directly proportional to the scenarios search space, which is a relevant aspect when dealing with timely exigent simulations. This conclusion facilitates the adaptability of the decision making process regarding the method's efficiency and effectiveness.

Additionally, when comparing the results of the proposed game theory strategy with the performance of two other well documented and reference strategies, it was found that this strategy is capable of achieving best results when the parameters are defined correctly. In fact, even when opting by a faster but less broad approach (parameterization with less considered scenarios and a smaller action domain), the game theory strategy was still able to achieve results in the same range as the reference strategies.

Considering the improvement of this method, further work will be done in what concerns a detailed analysis in the neighborhood of the scenario selected by the algorithm. To achieve this, an evolutionary approach will be included and combined with the game theory.

\section{Acknowledgments}

This work is supported by FEDER Funds through the "Programa Operacional Factores de Competitividade - COMPETE" program and by National Funds through FCT "Fundação para a Ciência e a Tecnologia" under the project: FCOMP-01-0124FEDER-PEst-OE/EEI/UI0760/2011. 


\section{References}

[1] Ahmadlou M. and Adeli, H., "Enhanced Probabilistic Neural Network with Local Decision Circles: A Robust Classifier,' Integrated Computer-Aided Engineering, 17:3, pp. 197-210, 2010

[2] Badaway, R., et al., "Agent-Based Coordination Techniques for Matching Supply and Demand in Energy Networks," Integrated Computer-Aided Engineering, 17:4, pp. 373-382, 2010

[3] Bandyopadhyay, S., "Multiobjective Simulated Annealing for Fuzzy Clustering With Stability and Validity," IEEE Transactions on Systems, Man, and Cybernetics, Part C: Applications and Reviews, 41:5, pp.682-691, 2011

[4] Baraldi, P., et al., "Genetic algorithm-based wrapper approach for grouping condition monitoring signals of nuclear power plant components," Integrated Computer-Aided Engineering, 18:3, pp. 221-234, 2011

[5] Ceppi, S. and Gatti, N., "An algorithmic game theory study of wholesale electricity markets based on central auction", Integrated Computer-Aided Engineering, 17:1, pp. 273-290, 2010

[6] Cristaldi L., et al., "Multi-agent Systems: an Example of Power System Dynamic Reconfiguration," Integrated ComputerAided Engineering, 17:4, pp. 359-372, 2010

[7] Dahabiah, A., et al., "Fusion of possibilistic sources of evidences for pattern recognition," Integrated Computer-Aided Engineering, 17:2, pp. 117-130, 2010

[8] Dore, A. and Regazzoni, C., "Interaction Analysis with a Bayesian Trajectory Model", IEEE Intelligent Systems, 25:3, pp. 32-40, 2010

[9] Federal Energy Regulatory Commission, "Notice of White Paper", U.S. Federal Energy Regulatory Commission, 4:28, 2003

[10] Federal Energy Regulatory Commission, "Report to Congress on competition in the wholesale and retail markets for electric energy", U.S. Federal Energy Regulatory Commission. Available: http://www.ferc.gov/legal/maj-ord-reg/fed-sta/ene-polact/epact-final-rpt.pdf

[11] Freitag, S., et al., "Recurrent neural networks for fuzzy data", Integrated Computer-Aided Engineering, 18:1, pp. 265-280, 2011

[12] Jing, Z., et al., "Study on the convergence property of re learning model in electricity market simulation", Advances in Power System Control, Operation and Man-agement, 2009

[13] Juang, C. and Lu, C., "Ant Colony Optimization Incorporated With Fuzzy Q-Learning for Reinforcement Fuzzy Control", IEEE Transactions on Systems, Man and Cybernetics, Part A: Systems and Humans, 39:3, pp. 597-608, 2009
[14] Koritarov, V., "Real-World Market Representation with Agents: Modeling the Electricity Market as a Complex Adaptive System with an Agent-Based Approach", IEEE Power \& Energy magazine, pp. 39-46, 2004

[15] Li, H. and Tesfatsion, L., "Development of Open Source Software for Power Market Research: The AMES Test Bed", Journal of Energy Markets, 2:2, pp. 111-128, 2009

[16] Neumann, J., et al., "The essence of game theory", IEEE Potentials, 22:2, 2003

[17] Operador del Mercado Ibérico de Energia, Polo Español homepage, http://www.omie.es, accessed on December 2011

[18] Pinto, T., et al., "Cost Dependent Strategy for Electricity Markets Bidding Based on Adaptive Reinforcement Learning", International Conference on Intelligent System Application on Power Systems - ISAP, 2011

[19] Praça, I., et al., "MASCEM: A Multi-Agent System that Simulates Competitive Electricity Markets". IEEE Intelligent Systems - Special Issue on Agents and Markets, 18:6, pp. 5460, 2003

[20] Prymek M. and Horak, A., "Multi-agent Approach to Power Distribution Network Modelling," Integrated Computer-Aided Engineering, 17:4, pp. 291-303, 2010

[21] Reuter, U., “A Fuzzy Approach for Modelling Non-stochastic Heterogeneous Data in Engineering Based on Cluster Analysis," Integrated Computer-Aided En-gineering, 18:3, pp. 281289,2011

[22] Santos, G., et al., "Complex Market integration in MASCEM electricity market simulator", International Conference on the European Energy Market 11 - EEM, 2011

[23] Shahidehpour M., et al., "Market Operations in Electric Power Systems: Forecasting, Scheduling, and Risk Management", Wiley-IEEE Press, pp. 233-274, 2002

[24] Tellidou, A. and Bakirtzis, A., "Agent-Based Analysis of Monopoly Power in Electricity Markets", International Conference on Intelligent Systems Applications to Power Systems, 2007

[25] Vale, Z., et al., "MASCEM - Electricity markets simulation with strategically acting players", IEEE Intelligent Systems Special Issue on AI in Power Systems and Energy Markets, 26:2, pp. 54-60, 2011

[26] Vale, Z., et al., "VPP's Multi-Level Negotiation in Smart Grids and Competitive Electricity Markets", IEEE PES General Meeting - PES-GM, 2011

[27] Wandekokem, et al., "Diag-nosing multiple faults in oil rig motor pumps using support vector machine classifier ensembles", Integrated Computer-Aided Engineering, 18:1, pp. 6174, 2011 